\title{
Designing Imperfection: The Semiotics of the Pixel
}

\section{Massimo Leone}

'Imperfection' is a key concept in both aesthetics and semiotics; Algirdas J. Greimas devoted a monograph to it (De l'imperfection, 1987), emphasizing the revelatory value of the aesthetic experience as capable to transport the subject from the appearance of imperfection to the perfection of being. Taking this elegant series of five semiotic analyses as a point of departure, the paper will seek to articulate a typology of imperfections in visual communication, arguing that each of them appeals to a different semiotic ideology and elicits, as a consequence, specific pragmatic responses. The article will indicate, in particular, some lines for the possible development of a 'semiotics of the pixel'. It is a highly speculative enquiry, for it must starts with a foundational skeptical question: are pixels semiotic objects at all? Do they signify autonomously from the image they give shape to? And if that is not the case, if they appear to be simple and inert constituents of digital configurations, what is their status then? According to Umberto Eco's witty definition, everything that can be used to lie can become an object for semiotic enquiry (1976: 18); but can pixels actually 'lie'? Or are they bound to produce light and color according to a cold mathematical rule, with no possibility for the randomness of intentional communication? (Mitchell 2005: 87-92). In other words: is it possible to design imperfection in digital signification and communication?

KEYWORDS imperfection, pixels, semiotics, aesthetics

'We look upon the geometric point as the ultimate and most singular union of silence and speech'. Wassily Kandinsky, Punkt und Linie zu Fläche (1926) ${ }^{1}$

1 Engl. trans. Howard Dearstyne and Hilla Rebay. 


\section{Introduction}

Semiotics has always inquired about visual signification, that is, about signs that predominantly elicit a reception of meaning by adopting signifiers that mainly work as perceptual stimuli for the sight, or that emulate or evoke such stimulation when they affect other senses or even that inner cognitive faculty of visual imagination that allows individual to conjure up, in their minds, mental constructs that closely resemble those which emerge at the actual empirical sight of images. Different perspectives can be adopted to study visual signification. They can be articulated depending on the specific consequences that such visual signification brings about: images can be studied as cognitive signs that encourage the formation of new ideas in the mind, as emotional signs that urge the transformation of a receiver's mood, or as pragmatic signs that prompt action toward a certain direction and with a certain goal. In exerting these effects, images often rely on the biological schemes of the neurophysiology of perception, which, nevertheless, are molded by sociocultural agencies into evolving patterns of visual culture or, rather, second visual nature.

An image, however, is not only something that has human beings think, feel, or act in a certain way. Images, and especially certain kinds of images, intrinsically and, sometimes, even impetuously trigger a meta-reflection on their very signification and meaning. Why do certain patterns of visual stimuli appear as such in the first instance, why do they stand out in relation to a context and present themselves as image, and why does this image signify, whereas analogous patterns of visual stimulation inconspicuously remain in the dark shadow of insignificance? Reflecting on such questions is not usual for semiotics, which has rather focused on the conditions and effects of signification of actual visual artifacts, but it is imperative for the development of its meta-semiotics, that is, a semiotically inspired philosophical inquiry into the very origins of visual meaning.

For instance, a mole manifests itself as a point or small area of the skin in which pigmentation is conspicuously different, and usually darker, in relation to the surrounding area. A mole can become an object of inquiry for semiotics. Medical semiotics, for example, can treat moles as signifiers signaling the potential presence of skin cancer whenever their visual configuration is altered in a specific way. Dermatologists can learn and master the code allowing them to infer, from the visual inspection of the texture of moles, the likeliness that the patient bearing them might be developing a melanoma. Similarly, a cultural semiotician may study the social schemes through which birthmarks, in a community of viewers, are interpreted as triggers of narrative hermeneutics, as it is traditional in many languages: called "voglie" in Italian and "alwahamat" in Arabic [meaning both "desires"], their color and sometimes also texture would originate from a pregnant mother's unsatisfied desire toward an object featuring the same visual qualities.

The cultural semiotician, however, should work hand in hand with the visual anthropolo- 
gist in seeking to determine the broad ethnological scenarios in which moles signify as they do. Called 'moedervlekken' in Dutch, 'modermærke' in Danish, and 'Muttermal' in German, birthmarks refer to a semiotic ideology according to which their epidermal imperfection, but also their potential as identity markers, ultimately derives from the mother, not from the father. In other cultures, such as the Iranian one, the origin of skin imperfection and identity is seen not as linked to a human origin, but as linked to a human agency in relation to a cosmological event: birthmarks, it is believed, would be caused by the fact that the mother has looked at the moon during an eclipse and has touched her belly (that is why birthmarks in Farsi are called 'lunar eclipses'). In other cultural contexts, like the Ethiopian one, a birthmark is seen as deriving from 'a kiss of Saint Mary the Virgin'. Cultural semiotics and cultural anthropology can and must relay these interpretations to more general and encompassing, albeit often invisible and surreptitious, semiotic ideologies of bodily imperfections.

There is, however, also another level of inquiry. Semiotics may not only rely a mole to a visual code or to a visual culture but also to that which could be called a 'visual temperament'. Why are moles significant in the first instance? And why are they visually received not only as visual evidence of a certain health condition (semeiotics) or as hints of a certain cosmo-genealogy (cultural semiotics) but also as triggers of a judgment of beauty, to the point that moles can be faked in order to provoke such judgment and, therefore, ipso facto become items endowed with semiotic relevance? In reflecting within this domain, the natural ally of semiotics is neither semeiotics nor cultural anthropology but aesthetics, considered as the philosophical speculation on the ultimate origins of the nature of feelings and sentiments of sensorial appreciation or depreciation.

Cooperation between semiotics and aesthetics has a long history: on the one hand, semiotics has provided traditional philosophical aesthetics with new frameworks so as to rethink, in the terms of a philosophy of signs, the immemorial problem of the definition of beauty. For instance, Umberto Eco's mentor at the University of Turin, Luigi Pareyson, pioneered a formalist understanding of the ontology of beauty and certainly influenced the later development of his disciple's visual semiotics (2013). On the other hand, scholars in the subfield of the semiotics of the fine arts inevitably interacted with preexistent aesthetic literature, either contrasting its approach polemically, for instance through promoting a anti-romantic understanding of the origin of beauty in the arts, or else drawing from aesthetics, and especially from the new trends of cognitive or empirical aesthetics, philosophical clues, and especially abstract questions, to be taken into account when debating about the signification of 'artistic' signifiers; Omar Calabrese's work in Italy, for instance, typically embodied both trends: a semiotically inspired aesthetics on the one hand, an aesthetically slanted semiotics on the other (2003).

The essay that follows is a contribution to this old and prestigious tradition of study, which has particularly flourished in Italy (Marrone 1990,1995) and, more generally, in relation to the semiotic attempt at understanding not only the meaning of artworks, but also the meaning 
of the artworks' meaning. In the task of developing, by semiotic means, a critical view of the contemporary digital aesthetics, the essay tackles a central question: may digital creation be conducive to a sense of beauty comparable to that which has been cultivated in pre-digital fine arts? More generally, is the visual texture of the digital suitable to trigger aesthetic appreciation? Both these questions fall in the traditional domain of aesthetics but could not be properly dealt with and, possibly, answered, without the contribution of a semiotic philosophy of artistic signification.

The essay will contend, in particular, that the human capacity of receiving a sense of beauty is intimately related to the potentiality of finding configurations of regularity and, therefore, meaning into visual patterns that appear, on the opposite, as swarming products of pure natural chaos. A tentative approach to this hypothesis is looked for in the semiotic and aesthetic comparison between two constitutive elements of the visual imagery: on the one hand, the point in pre-digital visuality and, on the other hand, the pixel as main component of the digital imagery.

\section{Pixel ubiquity}

We are surrounded by pixels. And we surround them. As soon as we wake up, we check emails, websites, and social networks on our mobiles; we interact with the LCD displays of appliances in our kitchen, bathroom, car; the screen of our computer 'talks' to us through patterns of pixels; when traveling, giant panels in airports and train stations communicate to us the times of arrivals and departures, pixel by pixel. Pixels are also the main components of digital craft; for instance, in animation movies; or even become the constituents of so-called 'digital art'.

Yet, despite their multifarious 'accomplishments', pixels remain 'hidden' to us, squeezed into the framework of a screen in increasingly high numbers, subserviently absorbed by the configurations of form, color, and brightness in which they inexorably disappear, compressed together by constantly improving technology so that their individual identity literally melts in the beautiful images that they compose.

The present essay is an attempt at indicating some lines for the possible development of a 'semiotics of the pixel'. It is a highly speculative enquiry, for it must start with a foundational skeptical question: are pixels semiotic objects at all? Do they signify autonomously of the image to which they give shape? And if that is not the case - if they appear to be simple and inert constituents of digital configurations - what is their status then? According to Umberto Eco's witty definition, everything that can be used to lie can become an object for semiotic enquiry (1976: 18); but can pixels actually 'lie'? Or are they bound to produce light and color according to a cold mathematical rule, with no possibility for the randomness of intentional communication? (Mitchell 2005: 87-92). 
Reflection on the 'semiotics of pixels' here will unfold in between two opposite perspectives, apparently distant and unrelated as regards their historical genesis and theoretical preoccupations. On the one hand, the enquiry will be inspired by one of the most famous classics of modern art theory, Wassily Kandinsky's Punkt und Linie zu Fläche: Beitrag zur Analyse der malerischen Elemente (1926). In particular, the present essay will tackle the question whether pixels might be considered as points, and whether pixels in digital art might assume the same aesthetic value as points in Kandinsky's 'metaphysics of graphics'.

On the other hand, the paper will reflect on pixels from the alternative framework provided by so-called 'parametricism': that is, the trend of aesthetic reflection that has developed from the adoption of parameters in digital art, and especially in digital architecture (Poole and Shvartzberg 2015). The two perspectives are, in a sense, diametrically opposite to each other, given that the former advocates a centrifugal aesthetic view on the pixel - turning it into the origin of a possible 'visual expansion' toward a horizon of autonomous signification - whereas the latter tends to turn the pixel into a pure numeric expression, whose semantic potential is completely predetermined by a series of set parameters.

Meditation on points and pixels (or on pixels as possible points of digital visual signification) will be conducted through an unconventional procedure, mainly by reflecting on a series of 'visual provocations' that compare pixels with similar aesthetic elements. At the same time, some grounding questions will constantly accompany the enquiry; in a nutshell, they are the following:

1. What is the difference between a pixel and a point?

2. Are there pixels in nature? And in fine arts?

3. Is digital singularity possible?

4. Is a semiotics of the pixel a viable project?

\section{Money, mirror, temple}

A good, provocative point of departure for rethinking contemporary aesthetics of the pixel is The Million Dollar Homepage, by Alex Tew (Fig. 1).

The story of this bizarre webpage is known to all digital art aficionados: impoverished British student Alex Tew had the idea to create a 1,000 x 1,000 pixel empty webpage and to sell the pixels on the internet in blocks, within a limited range of time, at the price of \$1 per pixel; pixels could then be used by purchasers as they pleased. The operation was an incredible, viral success: all pixels were sold within a few months, the last block being so coveted that an action on Ebay was organized. Alex Tew suddenly became a wealthy young man, for his funny idea was actually revealing, through provocation, an essential characteristic of the current digitalization of most contemporary visual culture. The rhetorical principles underpinning The Million Dollar Homepage can be summarized as follows: 
1. Pixels are offered in a limited amount;

2. Pixels are offered in a limited time;

3. There is no limit to the quota of pixels one can buy within the webpage;

4. There is no limit to what pixels may express;

5. There are no contextual constraints to what pixels may express;

6. There is competition for visibility (the bigger the better);

7. Competition requires the finite nature of pixels.

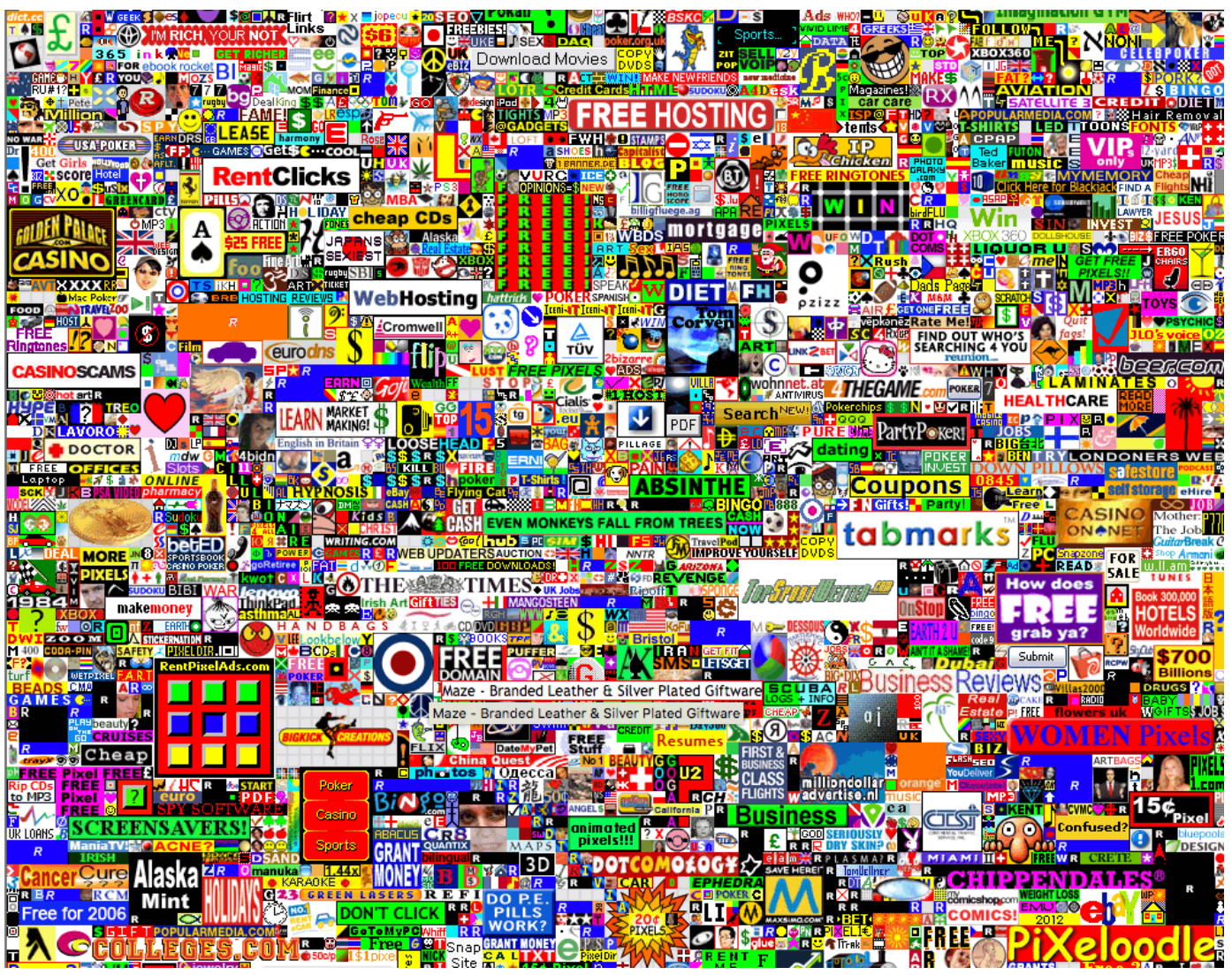

Figure 1. Alex Tew. As 8 February 2009. The Million Dollar Homepage. Webpage. 1,000,000 pixels; available at http://www.milliondollarhomepage.com/

The paradoxical nature of this remunerative provocation stems from the fact that pixels are, per se, infinitely producible: there is no limit to the amount of pixels that can be generated on the internet, meaning that there is no limit to the amount of different webpages that can be created, each one with a slightly different configuration of pixels. At the same time, the imposition of both a quantitative and a temporal framework triggers competition and, therefore, attributes social and economic value to something that is, at least in principle, valueless (Ma- 
trix 2006). But is that not the same dynamics through which most capitalistic discourse works? The imposition of a meta-frame inducing the idea of a limit of both resources and time ("the special offer') through an appropriate rhetoric fuels competition and stimulates the demand. René Girard's theory of mimetic desire explains well what happens in these cases: the more the pixels are desired, the more they become desirable (1977). There is no particular reason for which those pixels, and not others, should be sold, desired, bought; yet, the successful 'frame of valorization' created by Alex Tew turns the insignificant into significant, the valueless into valuable, the banal into the exceptional.

However, the experiment also reveals the social aesthetics of pixels from another point of view: purchasers pay not only for the possession of a certain quantity of digital figurative space but also for the possibility of using it in order to signify what they please. That is an essential characteristic of pixels, which, to a certain extent, makes them comparable with money: pixels are a protean matter, usable in order to give shape to any configuration of form, light, and color. First of all, buyers can express what they wish. In this regard, The Million Dollar Homepage unveils one of the most fundamental allures of the aesthetic economy of pixels: their value characteristically stems from the almost magical ability to grant their possessors the opportunity to 'express themselves', to use a certain amount and configuration of pixels as a matrix for voicing one's insatiable narcissism. From this perspective, pixels in Alex Tew's provocation do not work only like money but also like a mirror in which pixel purchasers indelibly imprint their visual identity.

Second, this manifestation of visual identity is not limited by any contextual constraints: not only may buyers signify what they want, they may do so also without any regard for the context; the purchase of a certain amount of pixels frees buyers to the obligation of 'digital sociality'. As a consequence, the principle of 'the bigger and brighter, the better' affirms itself: the vaster is the part of the page that digital egos occupy, the more they will be seen; competition for visibility, then, creates value and eventually even gives rise, at the end of the experiment, to an auction (the ultimate capitalistic mechanism for linking value and money).

At the same time, the frame invented by Alex Tew prodigiously turns the infinite nature of pixels into a finite one. Purchasers pay an extremely high price for something that a) actually exists in almost infinite quantities, like space or time; and b) actually does not exist apart from the display technology of the purchasers' own screens. On the one hand, The Million Dollar Homepage produces value exactly by artificially limiting in spatial and temporal terms the offer of available pixels; on the other hand, the webpage achieves its result by selling the idea that pixels will somehow be forced to signify their purchased content without limit of time and on every screen displaying The Million Dollar Homepage. This website therefore shows not only that pixels can be as fluid as money and as enticing as a mirror; it also shows that they can acquire the seductiveness of a temple, which often also selects a random portion of time and space (the perimeter of the sancta sanctorum) in order to turn emptiness into the sacred. The first person who drew the perimeter of a temple was a genius; the one who proposed an alter- 
native perimeter was a failure, or had to fight very hard to impose the alternative. Similarly, as the history of the Internet proves, Alex Tew's experiment could work only once.

\section{Elements and agency}

It is worth recalling, at this point, the etymology of the word 'pixel'. First appearing in a published document in 1965, 'pixel' is a contraction of 'picture element', literally, the element of a picture. A similar concept was expressed in German by the word Bildpunkt [literally, image point'], which appears in early patents related to the development of television technology, such as Paul Nipkow's 1888 patent. To be precise, though, a picture element and a 'picture point' are not exact equivalents (Paul 2016). Whereas the Bildpunkt can remind one of the point, metaphysically dealt with by Kandinsky in his abovementioned treatise, a pixel is, by definition, an element of something else; precisely, it is the element of a picture.

But what is an element in art? Can an artwork be seen as composed by such things as 'elements'? And is the point an element of art? Is the point an element of nature? And what is the difference between points in nature and in the pre-digital culture on the one hand and, on the other hand, pixels in the digital culture?

'Element' is a common word in several meta-languages, although usually associated with some sort of mechanical functioning; 'element' makes one think of the components of a mechanism or of a chemical compound. Metaphorically, its semantics can also be extended to cover the functioning of parts of non-mechanical whole; yet this extension is a priori limited in scope. Rarely will the word 'element', for instance, be used with reference to a limb of the human body, or to the sub-section of a novel, or, indeed, to the forms that compose a figurative artwork. What is the semantic rationale behind this difference? It probably consists in the fact that when something is called 'an element', this denomination implicitly affirms a diminution of its agency; an element of something is not something that expresses an autonomous intentionality and a self-centered agency but something that is subservient to the agency of a greater whole. That is the reason for which conceiving an artwork as composed of 'elements' or, vice versa, thinking that points, lines, surfaces, and colors are the 'elements' of an artwork, induces one to think that a static relation holds between the whole of the work of art and the parts that the (analytical) eye somehow discerns in it.

However, this use of the word and its semantic implications, as regards the functioning of an artwork, would be somehow misleading, suggesting, in fact, that such functioning ultimately is non-semiotic. On the contrary, claiming or reclaiming the semiotic functioning of the relation between the parts and the whole of an artwork means underlining that, while points, lines, and surfaces, together with colors and the general topological structure of the image, contribute to the overall aesthetic significance of the whole, they are not entirely subsumed by it and in it. They 
can continue to express an autonomous range of signification, which is nothing but the result of their capacity to exert an individual agency toward the eye of the spectator while somehow contributing this same individual agency to the coalescence of agencies that determines the final gestalt of the artwork. That is a first important difference between a point and a pixel; in Kandinsky's meditation on the visual language of western art, the point is never simply an element, but a monad that exerts both an individual visual agency and a collective visual agency, together with the other points, lines, and surfaces that appear in the graphic or pictorial work.

It is undeniable that the point is a component of visual and sometimes also non-visual artworks; yet most of Kandinsky's reflection about it consists precisely in underlining its autonomous and irreducible agency. That also explains the difficulty of defining the range of this agency and, with it, the topology of the point, which can be expressed only in the terms of a tension between the dimension of the point and that of the surface, as Kandinsky correctly suggested. The pixel, by contrast, is never a point but a square, or a rectangle in the case of some LCD curb screens; it differs from the point precisely because of this consubstantial morphology, which entails a whole series of aesthetic consequences. Differences between a point and a pixel are so sharp that they can be arranged in a scheme of structural oppositions. First, whereas in an artwork (even in a pointillist painting) each point is intrinsically different from the others, in a digital picture each pixel must be characterized by exactly the same potential, although this potential is subsequently actualized according to different parameters of color and brightness (Graw \& Lajer-Burcharth 2016). Second, whereas the characteristic circularity of the point makes it an essentially centrifugal eidetic component - expanding its visual agency in the surrounding iconic context - the pixel is characterized by a tetragonal structure, whose shape and technological functioning exactly aims at the opposite effect of turning the pixel into a centripetal visual element, unable to expand its visual agency onto adjacent pixels. In a point, color and brightness emerge and seek to irradiate the external visual space; in a pixel, by contrast, color and brightness are trapped within its squared perimeter, in which they must be activated or deactivated in total subservience to the program. A pixel that does not obey the overall program of the picture is not an element anymore but a malfunctioning pixel (Spieler \& Scheuermann 2012).

\section{Vibrating patterns, I: gravel}

It is instructive, at this point, to ponder similarities and differences between the functioning of the point in art and the functioning of similar morphological elements in nature.

Figure 2 reproduces a photograph of gravel on the path of Villa Casana in Novedrate, a university town in the proximity of Como; it was taken with the digital camera of a mobile phone in early October 2016. A conglomerate of objects that we would normally look at with 
indifference, engrossed in our daily routines, acquires a new aesthetic value once it is framed by the 'magical' rectangle of a picture and surrounded by a context that attracts the attention of the viewer toward its details. Gravel, then, ceases to be the inert material that we tread on our way to office and becomes a surface endowed with dignity, a beautiful tapestry composed of little multiform and multicolor stones, all arranged next to each other according to a mysterious and yet perceptible order. So as to complete the marvel of the observer, then, tufts of grass of different kinds - shining with several hues of bright green among the grey, white, and reddish little stones - emerge courageously from the gravel, adding a touch of organic asymmetry to its visual configuration.

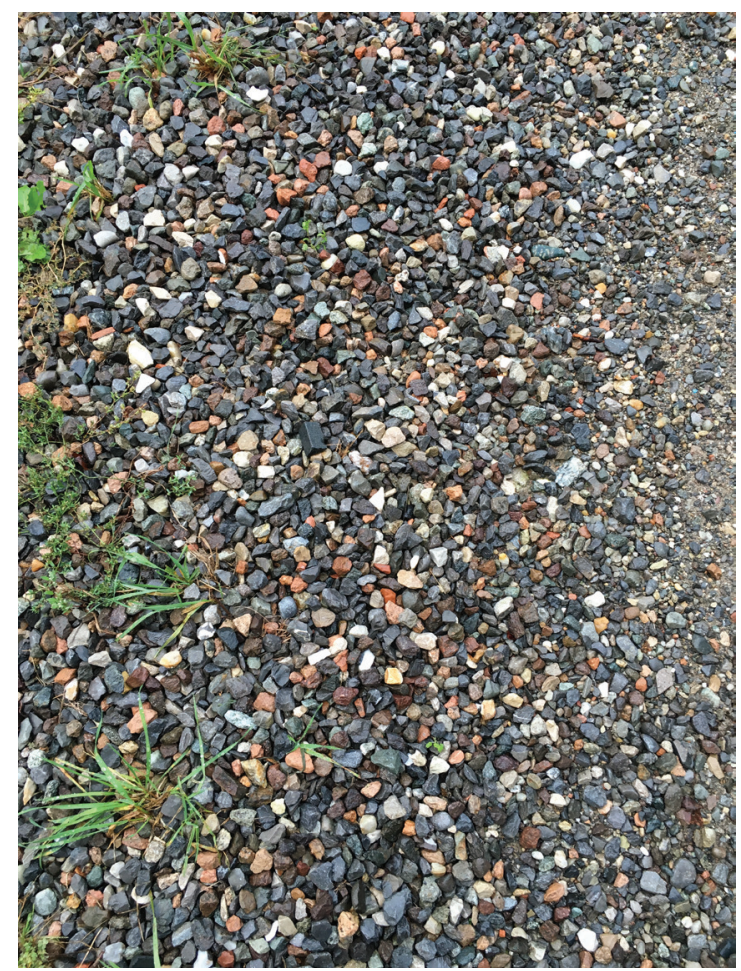

Figure 2. Early October 2016. Gravel on the path of Villa Casana, Novedrate, Italy. Digital picture (Iphone 6s) by the author

The aesthetic pleasantness of this visual gestalt, however, does not derive only from the superimposition of a frame; a holistic aesthetic principle seems to be at work herein: the juxtaposition of similar units creates more than their mere sum; a 'pleasant' aesthetic effect emerges from the juxtaposition. At a closer look, indeed, we perceive not only the little pebbles with their distinctive variety but also the whole that emerges from their complex arrangement; the interesting aspect of this aesthetic effect is that the two levels of perception and aesthetic agency interact and blur but, at the particular distance singled out by 
the photograph, never merge into each other: the observer can appreciate the individual qualities of the pebbles at the same time as this perception is somehow energized by the swarming gestalt of the gravel.

What is the origin of this aesthetic pleasure? Why should a visual configuration of this kind induce optical and aesthetic delight? Is it entirely subjective, or there is something objective in the pleasant tingling of the multitude? The key to understand the source of this optical pleasure seems to lie in the tension between similarity and difference, universality and particularity. It is also a matter of scale: as distance from the observer increases, heterogeneity yields to homogeneity. Should we look at the same gravel from a distance of one hundred metres, the perceptibility of its internal eidetic and chromatic difference would be dramatically blurred, the swarming effect of its texture dwindling until, in the end, only a greyish uniform surface would be perceived.

Symmetrically, looking closer and closer at the same configuration, smaller and smaller portions of it would be included in the view, until the visual focus would concentrate solely on a single pebble, or on a portion of it, and, therefore, be confined to the uniformity of one colored surface without morphological variations (these would reappear, of course, if an optical instrument enhancing human sight, like a microscope, was adopted). Uniformity, homogeneity, and indistinctiveness are the perceptual result of both a too distant and a too close gaze; in the middle, between these two opposite but actually adjacent polarities, lies the tension between a focus on similarity and a focus on difference, each a harbinger of a peculiar aesthetic pleasure. The 'holistic aesthetic pleasure' disappears when tension between similarity and difference is no longer perceptible. However, when it is, it is conducive to an oscillation between two cognitive as well as emotional conditions.

On the one hand, a close look entails an adaptive pleasure of aesthetic discovery: singularity 'hides' within universality; approaching the swarming gestalt of the gravel allows the observer to 'find out' what its visual components are; the individuality of pebbles is 'discovered' and almost 'rescued' in their multitude. Why should this pleasure be adaptive? It is not difficult to hypothesize that our ancestors might have benefited from an aesthetic condition giving them cognitive pleasure every time that they could 'look closer and better', in order to distinguish the traces of a predator in the sand, for instance, or the eyes of an enemy in a bush. The semiotic energy of the secret operates in this visual dynamics, wherein the particular, the individual, and the singular is as though 'hidden' in the general, in the collective, and in the plural: looking closer allows one to uncover a level of reality that is not immediately manifested.

On the other hand, a distant look loses this sense of individuality and progressively attributes more visual weight to the whole; the vibrating energy that it receives from its parts starts to dwindle and a feeling of compactness emerges; whereas a closer look to multitudes bestows upon the observer the sentiment of an aesthetic discovery, a more distant look en- 
tails a pleasure of totality, the bliss of a gaze that, suddenly, seems to encompass more of the reality and understands it better as a consequence. The pleasure of distinctiveness versus the pleasure of indistinctiveness: the two polarities imply diverging cognitive and aesthetic allurements but could not be appreciated as such if not within their mutual tension, that between the uniqueness of the object and its serial multiplicity.

\section{Vibrating patterns, II: sand}

In no other object is this tension as spectacularly visible as in sand. Dunes appear as uniform in color and internal morphology from a distance, but the vibration of the multitude of grains that compose them emerges as soon as one approaches one's gaze, a vibration that, then, diffracts into the surprising individuality of the grains of sand themselves as one looks even closer. Figure 3 reproduces photographs of grains of sands from different areas of the world; one of them is actually a specimen of sand from another planet (I will allow the reader the pleasure to find out which one).
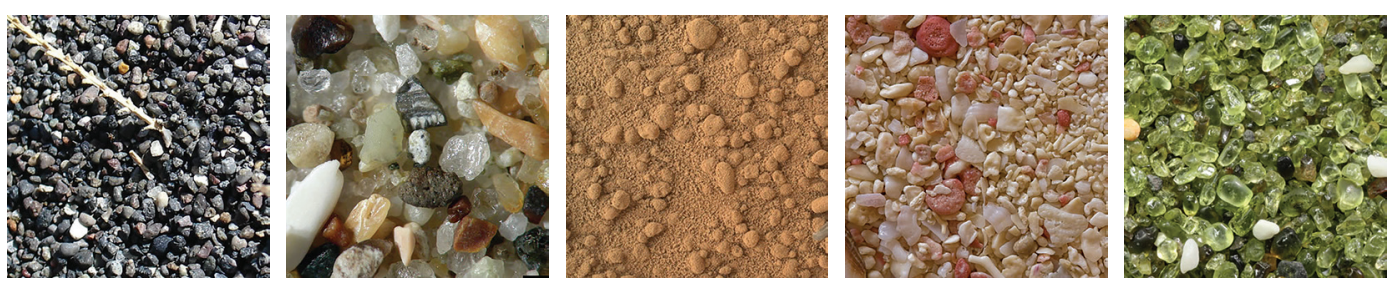

Figure 3. Photographs of different kinds of sand

Their chromatic difference is immediately evident. However, at a closer look, their eidetic particularity also becomes manifest. The grains in each picture appear as different from those in the adjacent photographs; moreover, even within each of the photographs, with an even closer look, individuality manifests itself as irreducible difference of shape, color, position and, as a consequence, texture. A closer look redeems the individual dignity of every single grain of sand. Grains do not cease to be part of a larger, vibrant whole, but acquire an almost personal beauty, as if they were each created by a dedicated agency.

A phenomenological look at the gestalt of sand makes one appreciate the emotional effects of closeness and distance: as one's gaze gets closer and closer to sand, for instance, particularity emerges in the form of irreducible singularity: each grain of sand is different; as one's gaze recedes, a different aesthetic pleasure, accompanied by a different emotion, becomes predominant: the world becomes more easily intelligible; the dune becomes predominant in perception. 


\section{Vibrating patterns, III: cobblestones and asphalt}

The dialectic tension between the singular and the plural as well as the aesthetic pleasure that it brings about manifest themselves not only in the observation of natural holistic configurations but also in that of human-made visual patterns. Figure 4, for instance, reproduces the photograph of a cobblestoned path in the Institute of Philosophy at the University of Leuven, Belgium.

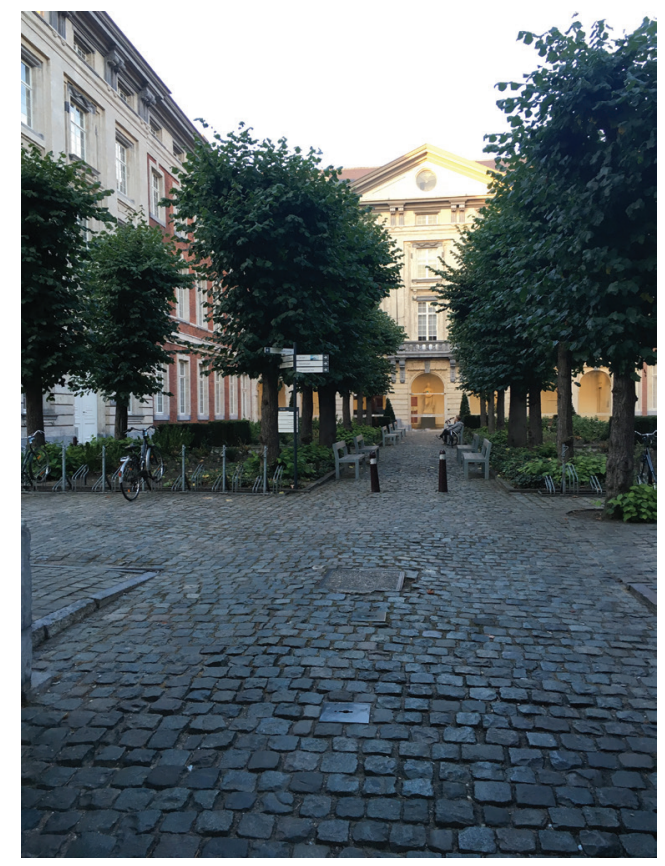

Figure 4. Late September 2016. Cobblestones at the Institute of Philosophy, University of Leuven, Belgium. Digital picture (Iphone 6s) by the author.

The material texture of the pavement is in part justified by functional purposes: for instance, increasing friction and traction in case of rain. This material texture, however, entails some aesthetic effects too: cobblestones are perceived as more 'pleasant' than asphalt. On the one hand, such aesthetic connotation is due to historical tradition and social conditions of perception: cobblestones are an architectural element that immediately sends the viewer back toward the past, when asphalt had not been invented yet or was not systematically used. As a consequence, cobblestones are now included in the common architectural alphabet of cities that want to rediscover their past, or rather reinvent it, and offer it to the aesthetic consumption of tourists and students. On the other hand, though, the pleasantness of cobblestones also derives from the intrinsic perceptual tension implied by their gestalt. That is not as extreme as in the case of gravel or sand, but it is all the more pleasurable as a consequence 
of that: at a certain distance, cobblestones appear as all very similar and orderly arranged in regular rows; this perception, however, never eliminates the underpinning aesthetic vibration, which is precisely due to small differences among cobblestones and to slight imperfection in their alignment (Uffelen 2009). The tension between the orderliness of the square - the homogeneity of the little forms, and their systematic layout on the one hand and, on the other hand, the persistence of subtle 'deformities', as well as minute varieties in color and topological disposition - turn this cobblestoned pavement into something that, to a certain extent, is alive, meaning that it presents perception with an internal visual tingling that is somehow akin to the movement of organic matter. To the gaze, this cobblestoned road surface swarms as though it was covered with big, square insects.

Other materials do not visually behave in the same way, mainly because of the different texture that characterizes them, a texture which, in turn, is a consequence of the physical structure of the materials. In this case, too, sociocultural connotations handed down through the history of the material interact with its intrinsic perceptual affordance. Were the road in the Institute of Philosophy of Leuven covered with asphalt, for instance, the enchanting melancholy that cobblestones signify and suggest to the observer would be replaced by an imaginaire of parked cars and futuristic efficiency.

Each material, though, has its poetry, and conceals in itself the organic aesthetics stemming from the dialectics between uniformity and deformity. It is just a matter of scale, and the right distance. It is sufficient to observe asphalt from closer, and with an affectionate eye, and then this apparently cold material, too, reveals in itself a beautifully disorderly world. Asphalt engineering looks at cracks in asphalt pavements as if they were mere problems due to the wrong fabrication or laying, and yet the typology of asphalt 'problems' reveals, to the eye of the attentive observer, a multitude of beautiful visual patterns, each traversed by a vibrant dialectics between the uniformity of the material and the chaotic tensions that explode through it (Field \& Golubitsky 1992). Figure 5 contains images of several types of 'asphalt pavement distress'; this technical term itself is interesting, as though asphalt, too, was able to 'suffer' and be 'in distress' like living beings are; the denomination of some forms of 'asphalt distress', then, like "fatigue 'alligator' cracking", for instance, implicitly hints at the fact that this inert material can, under certain conditions of transformation and at the right distance of observation, take on the gestalt of an organic texture, like animal skin:

Are not all these kinds of cracking 'beautiful'? Do they not look, when the frame of a picture surrounds them, isolates them, and invites the viewer to observe them from the 'right distance', like the visual configurations that contemporary artists so painstakingly seek to produce in their artworks? Could they not be the result of Alberto Burri's imagination? In each of these images, the dialectics between the reassuring uniformity of the material and the insurgence of asphalt grains reemerges, turning the material 'elements' of asphalt into as many sources of individual agencies, or at least into as many sources of agency that coalesce into independent 
subgroups. The fascinating aspect of this 'rebellion of the material' - as one could call it with an anthropomorphism - is that a micro-order seems to take shape within the disrupting disorder of the cracking. The cracking introduces deformity in uniformity, and yet this deformity too seems to be underpinned by a more complex formula.
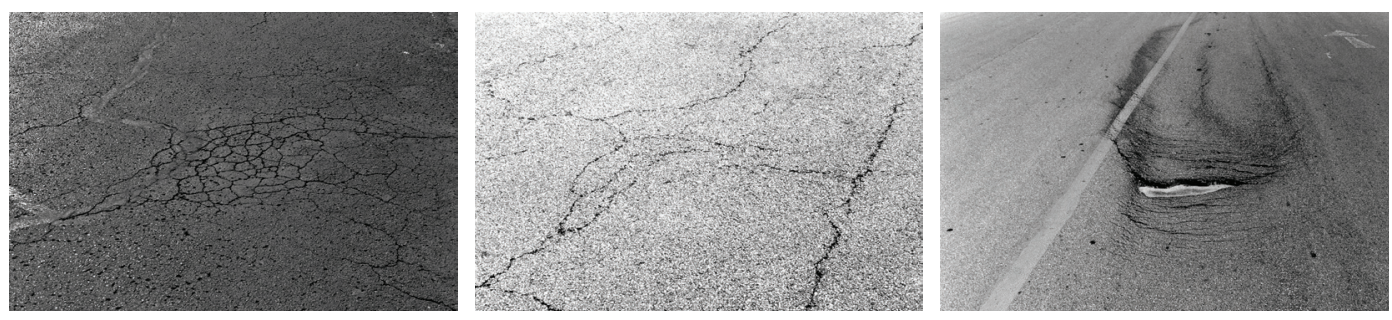

Figure 5. Different types of asphalt 'cracking': fatigue 'alligator' cracking, block cracking, and slippage cracking

\section{Vibrating patterns, IV: a flowerbed}

But what is the formula behind the holistic aesthetic effect that emerges from a picture like the one reproduced by Figure 6?

The 'holistic' aesthetic effect described above can be observed not only in the inorganic, mineral world (be it 'natural' or human-made) but also in the organic, vegetable world. Increase in morphological variety changes the characteristics of this effect; accrued presence of life often implies a more internally various gestalt; that entails extreme difficulty of representing the tension between homogeneity and heterogeneity; hundreds of species gather together in a single flowerbed; that reveals the inadequacy of botanical knowledge and representation. Botany can identify the plants and flowers of the flowerbed as tokens of botanical types, as members of a species, but does not take into account the singularity of each flower, of each plant. This flowerbed exerts an almost hypnotic optical power exactly because its overall gestalt emerges from the juxtaposition of a very high number of singularities.

As it was suggested earlier, there is a relation between life and increase in the morphological complexity of a multitude. If life quintessentially coincides with movement, this flowerbed differs from a cobblestoned pavement precisely because its internal visual structure is constantly vibrating under the effect of a myriad of motilities. As a consequence, a Botticelli, or another extremely skillful artist, would be required in order to render the beauty, but also the morphological intricacy, of hundreds of species competing or cooperating for life not only as a species, but also as individual members of that species. The more one moves forward through natural evolution, the more one comes across species endowed with the ability to accomplish elaborate movements with increasingly higher degrees of freedom and complexity. Were a 
horse pasturing on this flowerbed, for instance, the very wide range of movements that the animal could easily perform would further complicate the visual intricacy of the scene. A Michelangelo would be needed to render this holistic visual dynamics in a single static pictorial image.

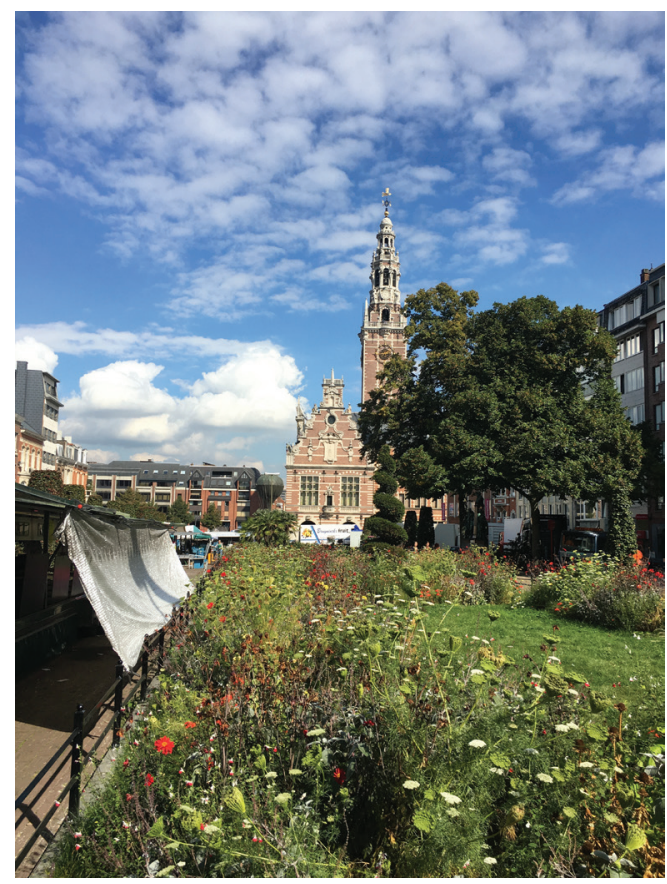

Figure 6. Late September 2016. Flowerbeds at the Public Library, Leuven, Belgium. Digital picture (Iphone 6s) by the author

One might hypothesize that the tension between homogeneity and heterogeneity that, at the right distance, a landscape of dunes or even an asphalted road manifest to the gaze, is in proportional relation with the degree of freedom of movement and, therefore, change, that the elements of these visual gestalts can enjoy. As geology suggests, minerals change through time too, and yet the speed of this change is mostly invisible to human perception (except in spectacular phenomena like earthquakes or volcanic eruptions); the interplay between sand and wind makes the former much more subject to movement and change than a rock or a cobblestoned pavement might be (indeed, cobblestones and other pavement materials have been invented or adopted exactly in order to limit this mutability and its uncontrollable and undesirable effects: it is easier to travel on cobblestones than on sand). The complexity of this interplay of agency results in visual effects at the macro-level of the gestalt of dunes, which do not appear as immobile blocks of sand grains but as movable, almost living, beings. A flowerbed, or, even more, a flowerbed on which horses graze, is a place in which this interaction of agencies, and the aesthetic effect resulting from it, becomes exponentially more complicated. 
Our gaze is pleased by a flowerbed because it is intrinsically a spectacle of freedom, in which myriads of competing agencies nevertheless find a way to harmoniously occupy the same time and space (Tao 2008). Incidentally, that explains the difficulty of depicting a battle scene. The great pictorial representations of battlegrounds in art history all look like flowerbeds, for their static character - as well as their intrinsic aesthetic aim - does not allow the painter to sincerely express the chaos of a battle; the depiction of a battle is always somehow harmonious, as though the warring agents would nevertheless agree in assuming their postures, forms, and colors in order to fit within the frame of the painting.

\section{Vibrating patterns, V: textiles}

The pleasant aesthetic effect of the interaction between idiosyncratic agencies and their holistic harmony results from hand-made visual configurations too. The cobblestoned pavement was an example of it, although in that case its aesthetic effect was certainly less predominant than its practical purpose. In any case, the aesthetics of a cobblestoned pavement is generally unintentional, or follows the aesthetic inertia of architectural fashion. There are, in contrast, human artifacts that explicitly seek to exploit the dialectics between singularity and universality for aesthetic purposes. Many forms of weaving craft, for instance, consist in the juxtaposition of knots or other weaving units that, although composing a regular pattern and sometimes even a complex figuration (as in tapestry, for instance), maintain, nevertheless, their subtle individuality and, therefore, contribute to the unpredictable vibration of the whole. Take, as an example, the Equatorian alpaca carpet from Otavalo market whose photograph is reproduced in Figure 7:

Taking a closer look, each of the knots that compose this carpet is different from the others. Without this singularity, the texture of the rug, but also its tactile affordance, would be completely different. Within a certain range of observation distance, the carpet looks as it does exactly because it constantly proposes the interaction between two phenomenological levels: on the one hand, the vibration of a multitude of individual knots, each slightly different from the others in terms of color, topological position, as well as internal morphology; on the other hand, the emergence of an harmonious but vibrating gestalt from the juxtaposition of these singularities; the individual agency of the knots is not completely eliminated (the fibers that compose the knots twist and turn, each with a peculiar bend in space and time), and, yet, it is somehow curbed within the overall gestalt of the rug. This subtle dialectics affects not only the visual configuration of the carpet but also its tactile affordance. Stepping barefoot on such carpet feels like it does because the knots in it have an internal structure and, to a certain extent, a freedom of movement that molecules of marble in a marble pavement do not have. The material tactility of a carpet is also an expression of the degree of freedom of its internal morphology. 


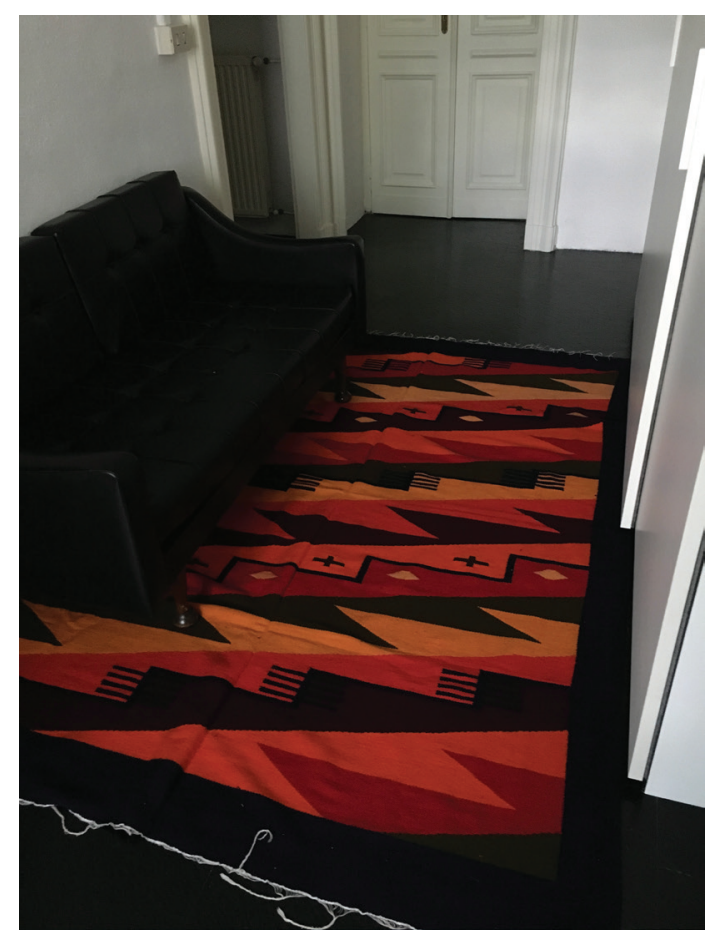

Figure 7. Equatorian carpet from Otovalo market. Digital picture (Iphone 6s) by the author

Ghiordes (symmetrical) and senneh (asymmetrical) knots; jufti (over four warp threads) and Tibetan knots: there is not only a large variety of knots (Fig. 8) but also an individuality of them, for they are made by hand; as a result, the aesthetics of hand-made rugs is different from that of machine-made rugs. The variety of the internal morphology of a rug is increased by the possibility to choose among different kinds of types of knots, exactly like the internal visual variety of a flowerbed depends on the possibility of choosing among different species of plants and flowers; however, a hand-made rug somehow imitates a flowerbed also because its internal morphology vibrates as a consequence of the fact that no knot is exactly like the others, in the same way as each rose shows micro-morphological peculiarities while belonging to the same species as all other roses (Bahamón and Pérez 2008). The hand of the rug-maker strives for perfection, and yet its intentionality translates into movements that are never completely standardized. Fatigue, state of mind, conditions of work change throughout the hours, the days, the months, the years, thus introducing slight perturbations in the movement of the hand that knots a rug. The unpredictability of the human hand's agency somehow transfers into that of the knots of a rug, exactly like a mysterious variation is constantly introduced into the morphology of rocks, animals, and human beings. 'Creating' a hand-made rug is different from 'fabricating' one by machine, because the former operation reminds one of the unpredictable creativity of nature, whereas the latter seems awkward in its dealing with the tension between perfect universality and idiosyncratic singularities. 

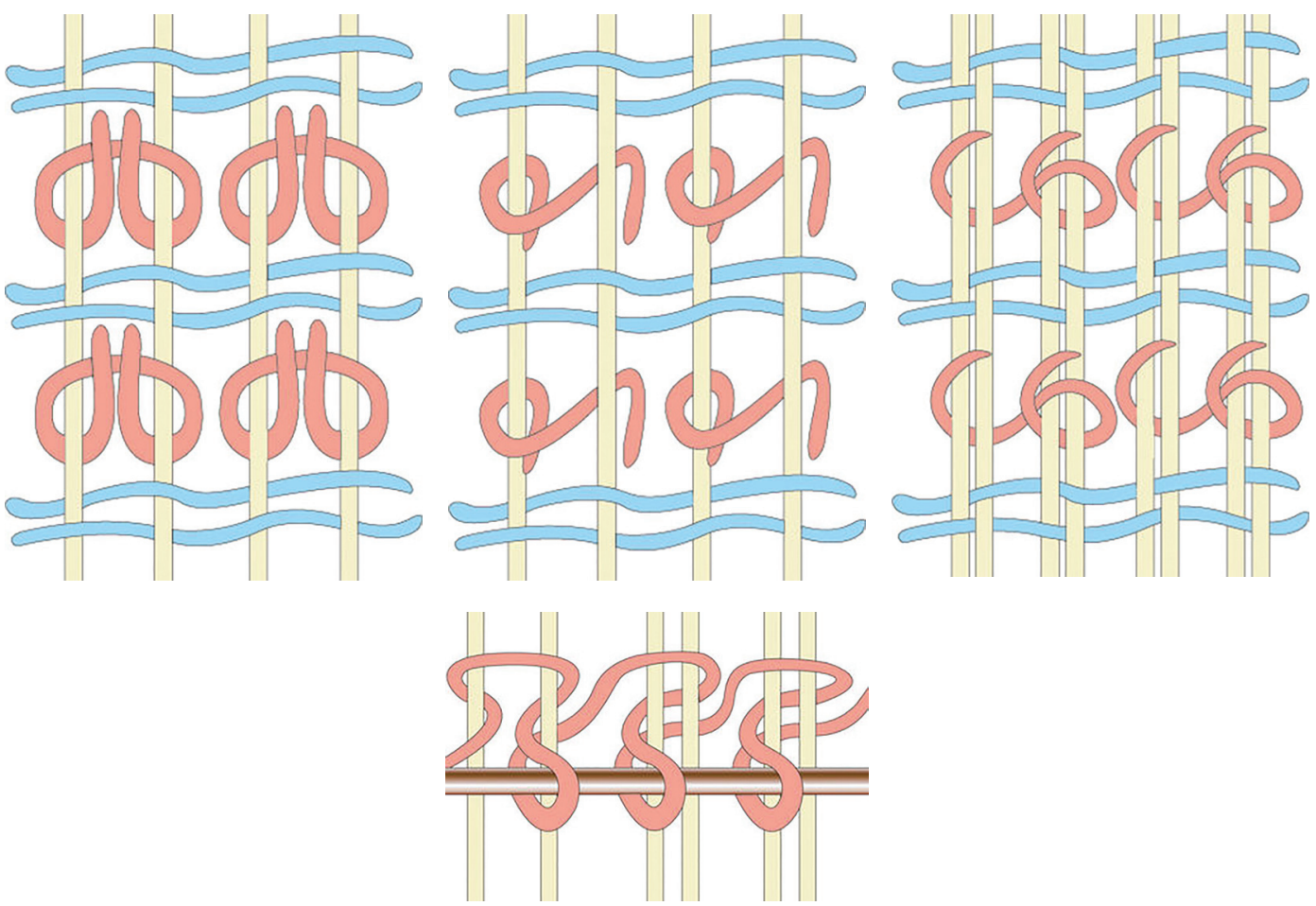

Figure 8. Types of rug knots
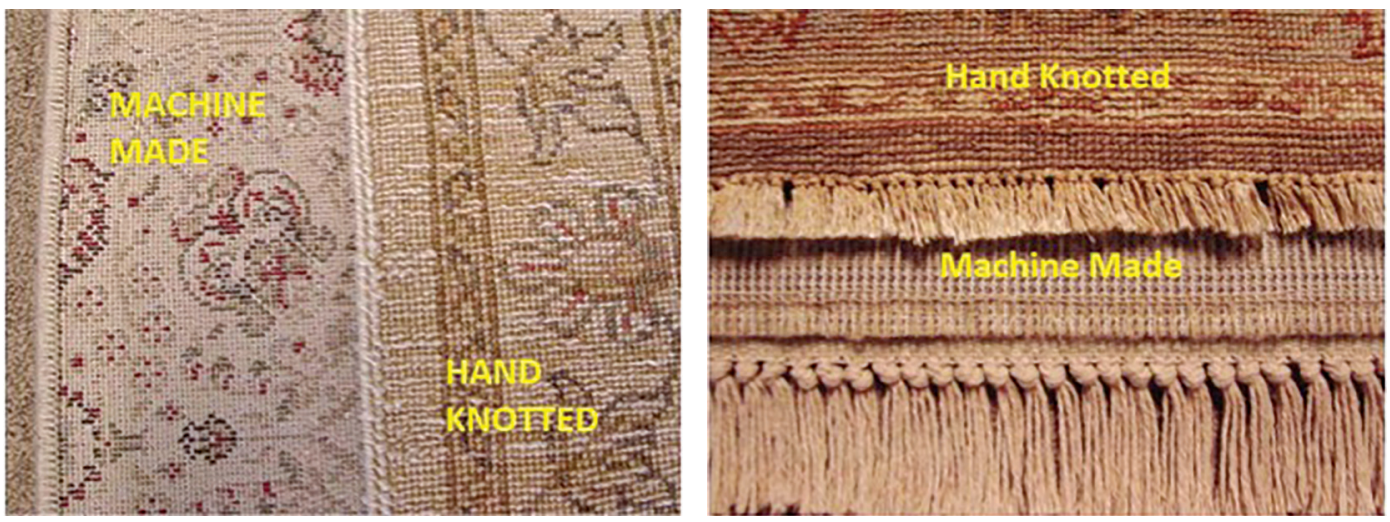

Figure 9. Differences between hand- and machine-made rugs

The comparison between the gestalt of a machine-made rug and that of a hand-knotted one reveals the paradoxical dialectics between perfection, imperfection, authenticity, and aesthetic quality: an authentically hand-made rug is knotted so as to minimize imperfections; yet, imperfections persist and constitute the uniqueness of the rug, as well as its value and mark of authenticity; machine-rugs are less expensive because they are too perfect. Fake imperfections intentionally introduced in rugs by human rug-makers tend to be stere- 
otypical, but more creative than intentional, machine-produced imperfections. The mastery of rug-making, which is based on the mastery over knots, consists in the ability of arranging the potential motility of warp threads into regular patterns; this reduction of complexity is never exhaustive, and that is exactly what makes the difference with a machine-made rug. In a hand-made rug, the regularity of knots constrains their material singularities; yet these singularities, and the agency they evoke, never cease to vibrate under the surface of the visual pattern, as if they were tiny servants constantly on the verge of bursting into a rebellion. In general, the tension between the struggle to perfectly subdue the idiosyncrasy of matter and the constant possibility of an emergence of imperfection seems to generate an aesthetic pleasure that is akin to that which is enjoyed by the beholder of a dune, of a flowerbed, or of a cobblestoned pavement, but enhances even more the dialectics between individual agencies and overarching gestalt. Fig. 10 reproduces the photograph of a Panama hat recently bought in Ecuador.

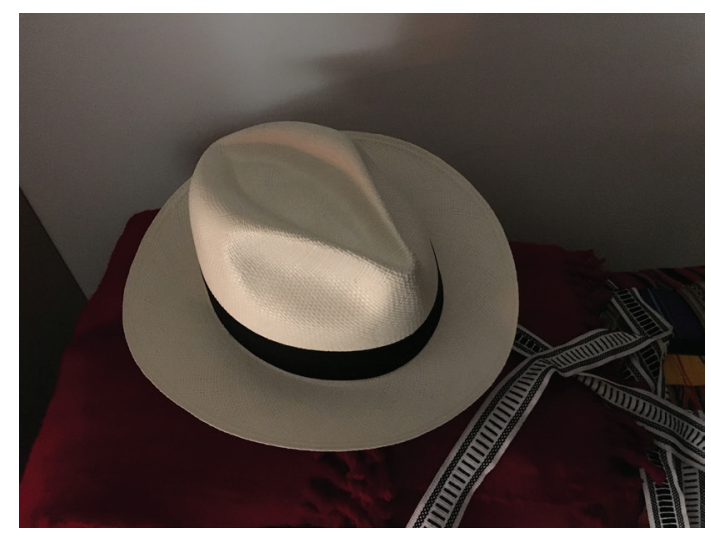

Figure 10. Mid-September 2016. Equatorian Panama-style hat, Quito, Ecuador. Digital picture (Iphone 6s) by the author

At this stage of the article, it is quite easy to observe that, in this hat, too, when it is looked at from an appropriate distance, a dialectics between singularity and universality, heterogeneity and homogeneity takes place. Mutatis mutandis, a Panama hat aesthetically works like a rug: in a limited amount of space, determined by the framework of the hat's shape, single compositional units must be juxtaposed and woven together so as to fill and at the same time create the form. In this case too, the manual work of the hatter strives to reduce the idiosyncrasy of the straw fibers, to subdue them into a regular order; yet, each fiber 'behaves' both morphologically and chromatically in a peculiar way, yielding to the general scheme followed and enforced by the hatter, yet constantly threatening to escape it. As a consequence, the threat of small imperfections makes an authentic panama hat vibrate with the dialectics between the plan of human creativity and the resistance of matter. Differently from a Persian 
rug, though, this plan does not involve bi-dimensional figuration but three-dimensional shape. Straw fibers must give rise to the shape of the hat.

\section{Hats and screens}

So as to gradually return to a 'semiotics of the pixel', it might be interesting to wonder: what is the difference between buying a new digital screen and buying a new Panama hat? First of all, how does one assess the quality of a Panama hat?
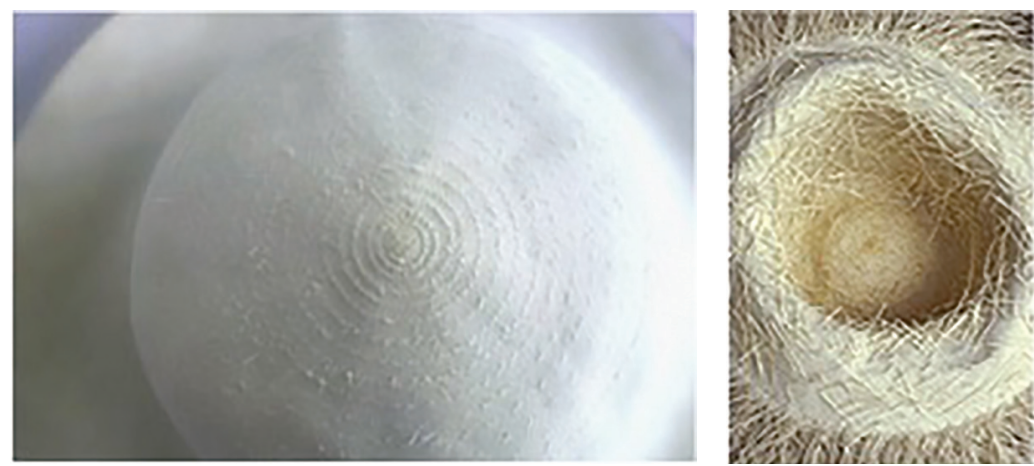

Figure 11. Rings inside a Panama hat

On the one hand, the quality of a Panama hat depends on some objective, countable features, such as the number of rings inside the hat or the number of straw fibers per inch. Figure 11 reproduces two photographs of these countable signs of the quality of a Panama hat. They are functional qualities too: a tightly-woven Panama hat will be more resistant, resilient and, in general, capable of maintaining its shape against the hostile agency of external forces (rain, wind, someone sitting on the hat, etc.).

On the other hand, though, the quality of a Panama hat also depends on some inter-subjective, uncountable features: the shape of weaving, for instance; or the color of straw.

Panama hat connoisseurs usually prefer the straw weaving reproduced in the second of the photographs of Figure 12, praising its impeccable regularity (at least at this distance of observation), in contrast with the irregularity of the first specimen. However, this time the criterion at the center of the evaluation is not objective, meaning that there is no quantitative, countable way to determine which straw arrangement is better, which one is worse. Stereotypical aesthetic evaluations in this domain form as a consequence of the very complex and mostly implicit negotiation within a community of interpreters guided by a group of experts. 
As a result of these negotiations, Panama hats with regular arrangements of straw fibers become part of the canon and are sold at higher prices, whereas Panama hats with irregular fiber arrangements are less favorably judged and less expensive. A simple question to ask in order to distinguish between countable and uncountable criteria of aesthetic evaluation, between objective and inter-subjective standards is: will it change in time and space? As regards the number of straw fibers woven in a Panama hat, the answer is negative: one day we might value a loosely woven Panama hat more than a tightly woven one, and yet the former will always have more fibers than the latter in the same amount of space; in this domain, its superiority will always be measurable. In the second case, instead, the answer is positive: one day, the community of interpreters that evaluate the quality of Panama hats might change in such a way that hats with irregularly arranged straw fibers might look more 'authentic' than hats with regular fibers (for instance, as a consequence of the massive diffusion of splendidly woven machine-made hats). That is even more evident as regards color: there is no objective reason for which one of the straw fiber colors represented in Figure 13 should be valued more than the others.
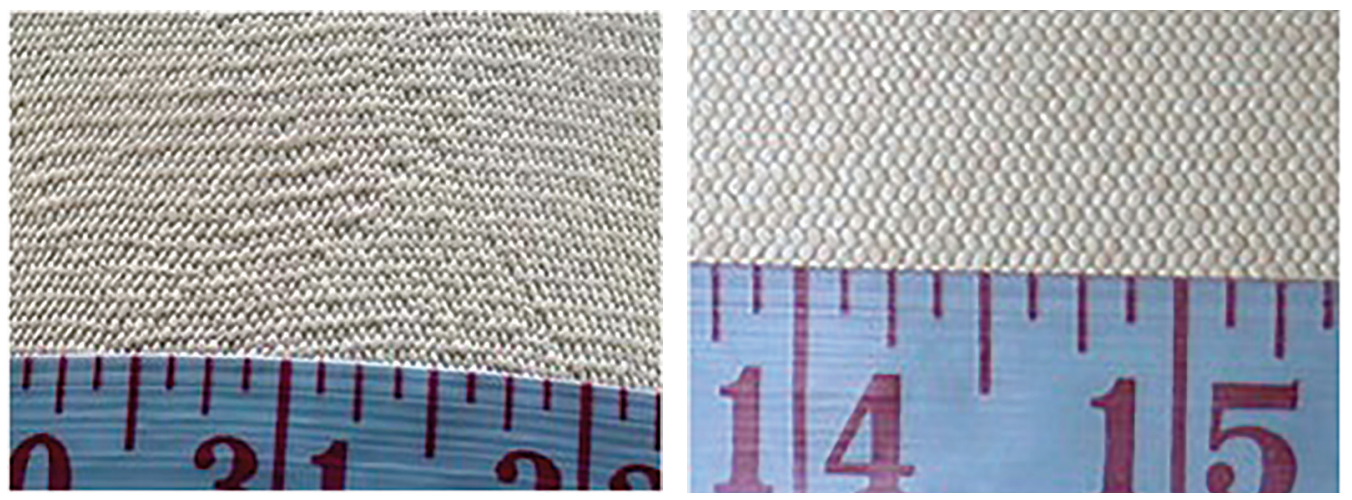

Figure 12. Regular and irregular straw weaving patterns in Panama hats

Whereas the first criterion is a quantitative one, the second is a qualitative one. It does not bear on a perception that can translate the qualities of a phenomenon into quantifiable measures and, ultimately, comparable numbers. It bears on an aesthetic appreciation that, again, is able to receive aesthetic pleasure from the dialectics between uniformity and deformity, between the smooth surface of a Panama hat and the subtle imperfections that make its texture vibrate at both sight and touch. A Panama hat is a result of creation as much as a rug 
is. They both 'imitate' the complex way in which natural agencies and, ultimately, life, give rise to a sand dune, or to a flowerbed. They reproduce, in their internal morphology, the struggle between idiosyncratic mutation and collective order that seems to characterize the patterns of evolution (Romero and Machado 2008).

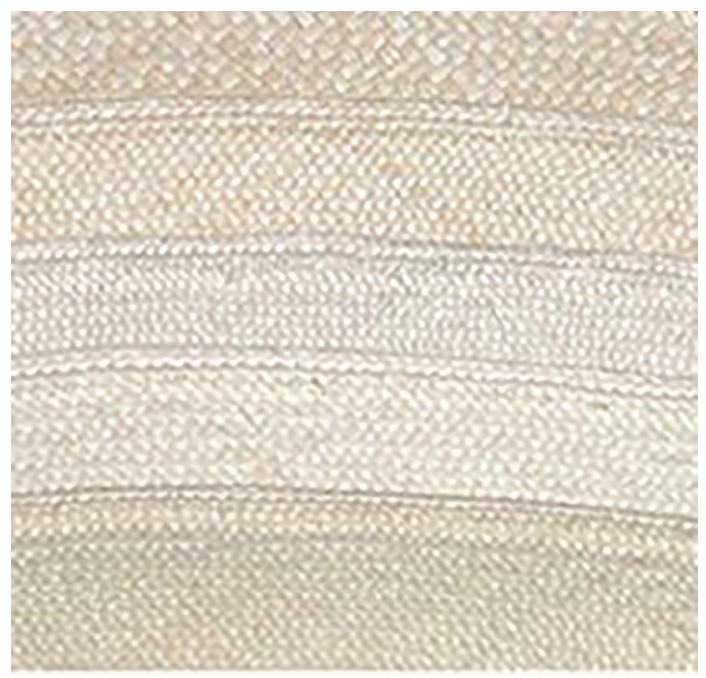

Figure 13. Colors of straw in Panama hats

\section{Deconstructive pleasures}

Figure 14 reproduces the photograph of a tapestry currently hanging in a boutique hotel of Quito, Ecuador. From a semiotic point of view, a tapestry is a carpet in which the plastic level is 'forced' to express a figurative level. However, the plastic level of a tapestry never completely disappears but contributes to the aesthetic effect of the figurative level. In a tapestry, a constant plastic vibration underlies images. The quality of this 'vibration', though, changes depending on how the tapestry is woven. The way in which the single knots are knotted, and give rise to the overall figure, influences its perception by the beholder.

The same principle is observable, with different modalities, in everyday decorative arrangements, such as the one of the fruit-salad of Figure 15:

There are no compelling functional reasons by which fruit should be cut and presented in this way (except, perhaps, making it easy to pick them with a fork or spoon). The aesthetic quality of such arrangement derives, again, from the pleasure that human beings feel in deconstructing the figurative level of reality into combinable ingredients, such as the colorful geometric shapes of this fruit-salad. As in a rug, or in a tapestry, or in a Panama hat, the idea that tiny units might coalesce into an overall effect of shape and sometimes even figure is, for 
some reason, enthralling. In a fruit salad, human beings play with the dynamics of order and disorder, homogeneity and heterogeneity, deconstruction and reconstruction that underpin the natural world and its perception.

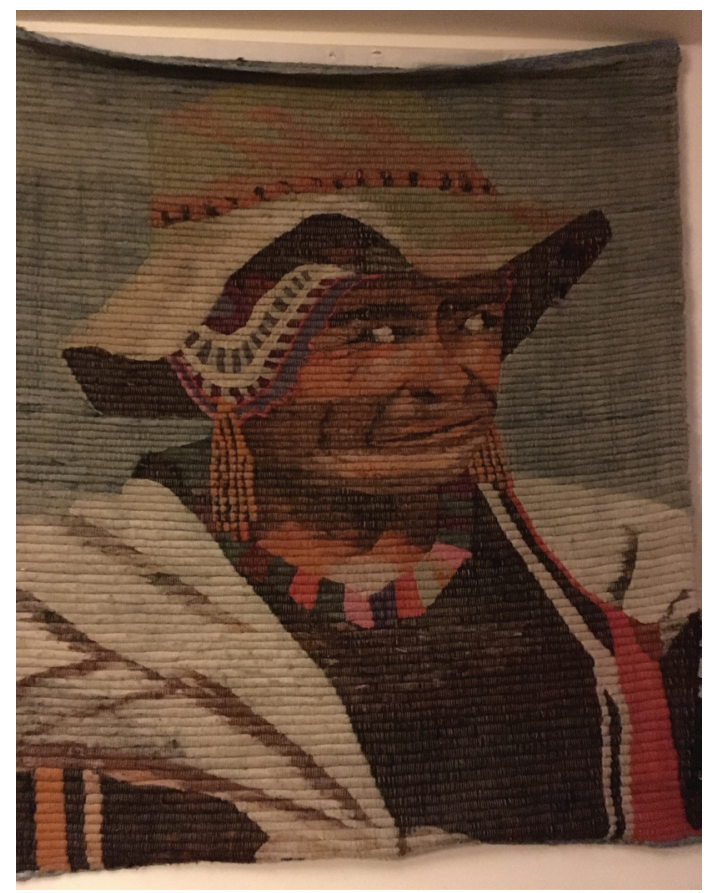

Figure 14. Mid-September 2016. Equatorian tapestry, Casa Joaquin Boutique Hotel, Quito, Ecuador. Digital picture (Iphone 6s) by the author.

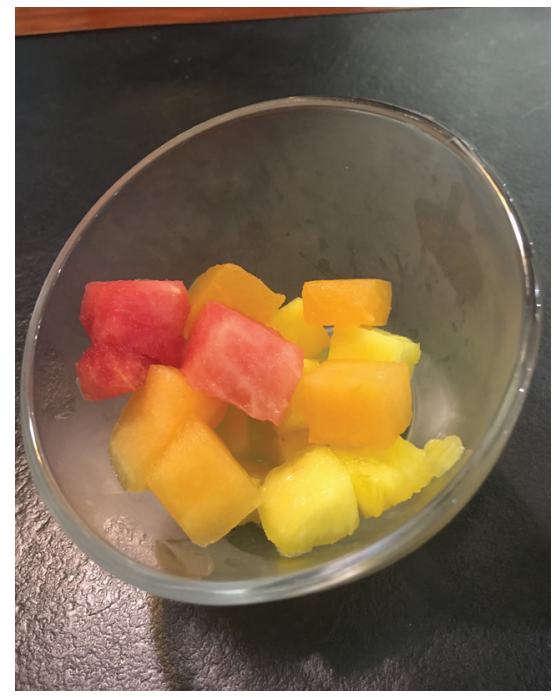

Figure 15. Fruit salad 


\section{The aesthetics of pixels}

Is the same dialectics clearly visible in LCD screens too? Is the quality of such objects gauged in the same way as that of Panama hats is, by conflating countable and uncountable criteria of evaluation? Does a digital screen decompose and recompose an image of reality in the same way as a rug or a mosaic do it? And, last but not least, is a screen, too, the visual framework of a creative production of icons, reminiscent of the way in which nature itself manifests its visual morphology in minerals, plants, and animals?

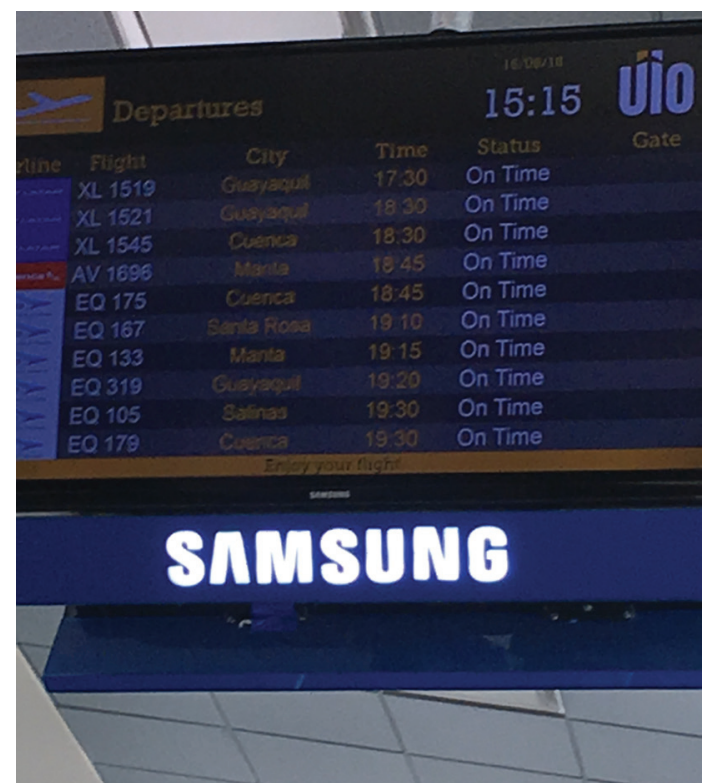

Figure 16. Mid-September. Giant screen at Guayaquil Airport, Guayaquil, Ecuador. Digital picture (Iphone 6s) by the author.

Figure 16 reproduces the photograph of a giant screen displaying arrival times at the airport of Guayaquil, Ecuador. Similar screens have been installed in almost every airport or train station around the globalized and cosmopolitan world. Is the aesthetic functioning of this LCD screen somehow comparable to that of the natural patterns or the visual artifacts that have been mentioned so far? When comparing several digital LCD screens among them, the only thing that really matters is a countable criterion, that is, the number of pixels that are squeezed within the framework of the display. Indeed, there cannot be an alternative criterion, for pixels actually are elements, as the etymology of their name suggests. They completely abdicate any autonomous aesthetic agency in order to become totally subservient to the project of the overall picture. They are not round - a feature that Kandinsky would deem essential in the visual metaphysical force of the point - but square or, at the most, quadrangular, like in the most recent curb LCD screens. Most importantly, they do not express any singularity, 
not simply because their shape is always exactly the same, but also because their value of brightness and hue can be determined by a numeric value, with no margin for idiosyncrasy. A pixel that does not abide by the mathematical rules of the figurative project of which it is an element is a malfunctioning pixel.

Pixels, therefore, do not aesthetically function like grains of sand or cobblestones because their individuality is thwarted at the very moment of its fabrication. They also starkly differ from the knots of a rug or the tiles of a mosaic, not only because of their lack of 'deformity' and, therefore, singularity, but also and above all because the enunciation of a pixel must coincide with the disappearing of it. In a carpet, the dexterous hand of the rug-maker has juxtaposed very tiny knots so that they might almost melt into elaborate figurative patterns; yet, the individuality and singularity of knots, as it was underlined earlier, persists, and contributes to the aesthetic effect that this paper has singled out and described both in nature and culture.

In contrast, the possibility of a digital art of the screen is somehow limited by the way in which its constituents, the pixels, are hidden from sight and reduced to total obedience - for such is the quantitative promise of the market to LCD purchasers (Castelli and Berliner Festspiele, Martin-Gropius-Bau 2007). The rhetoric of pixels does not reveal their semiotic nature but their robotic perfection, their cold splendor, Lévinas would have said. Whereas paintings, tapestries, rugs, and even Panama hats retain something of the liveliness of the face, with its dazzling vibration of imperfections, the LCD screen is a façade, a surface that, like a mirror, divests itself of any semiotic functioning in order to yield to the reassuring regularity of a mathematical ratio. An image displayed by an LCD screen appears as perfect to the gaze, even more perfect than its content does in reality to sight; and yet, this content is somehow deprived of life, since it is stripped of that dialectics between the regular and the eccentric that underpins most beholding of a visual multitude in both nature and culture. That does not mean that LCD screens are intrinsically unable to reveal this dialectic. In this regard, a semiotics of flickering is in order. Lagom LCD monitor test pages define this instance of technological idiosyncrasy:

In a pixel on an LCD monitor, the amount of light that is transmitted from the backlight depends on the voltage applied to the pixel. For the amount of light, it doesn't matter whether that voltage is negative or positive. However, applying the same voltage for a long period would damage the pixel. For example, electricity decomposes water into oxygen and hydrogen gas. A comparable similar effect could happen inside the liquid crystals that are in the pixels. In order to prevent damage, LCD displays quickly alternate the voltage between positive and negative for each pixel, which is called 'polarity inversion'. Ideally, the rapid polarity inversion wouldn't be noticeable because every pixel has the same brightness whether a positive or a negative voltage is applied. However, in prac- 
tice, there is a small difference, which means that every pixel flickers at about 30 hertz. (Available at http://www.lagom.nl/lcd-test/inversion.php)

It is extraordinary how the flickering of light bulbs or, more recently, of LCD screens stereotypically intervenes in the climactic moments of horror movies. Flickering, indeed, signals the arrival of the uncanny, the monstrous, and the horrific through the idiosyncratic malfunctioning of that which, by definition, should be as reliable as a machine. Flickering, therefore, signifies the manifestation of a mysterious, often malign agency in a mechanism that should not have one. All of a sudden, the pixels of an LCD screen cease to be picture elements - subservient to the cold perfection of the image they are supposed to give shape to by renouncing any individuality - and turn into Quixotic mavericks, possessed by a dangerous force.

The flickering of LCD screens (Fig. 17) is the digital equivalent of the malfunctioning of flap displays, less and less in use around the world and almost entirely replaced by digital screens. However, the latter would entail a strong narrative dimension - a sort of suspense - and deploy an aesthetics of imperfection that was heavily dependent on the essentially mechanical nature of the display (Hagener and Hediger 2015) (in Fig. 18, a split flap display in the arrival area of the airport of Belgrade, Serbia, with 'Dadaist' malfunctioning).
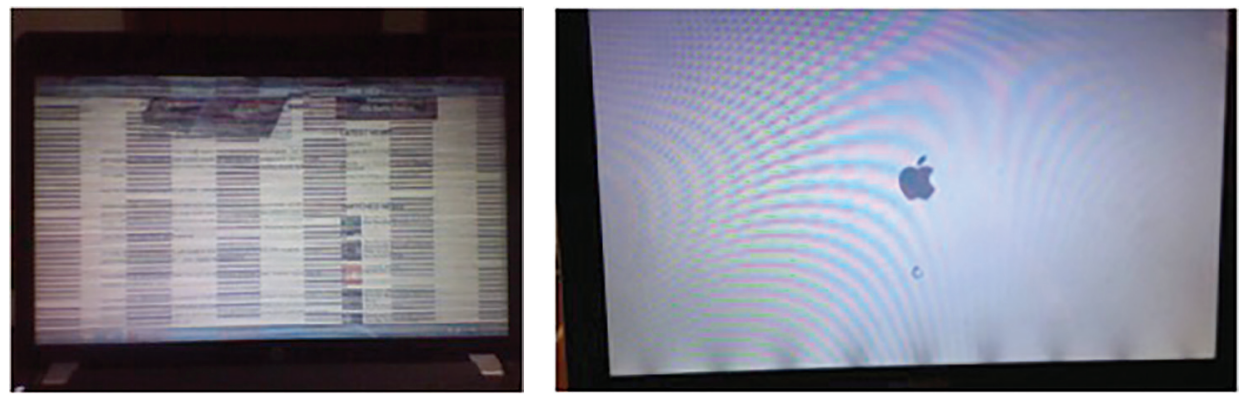

Figure 17. LCD screens flickering

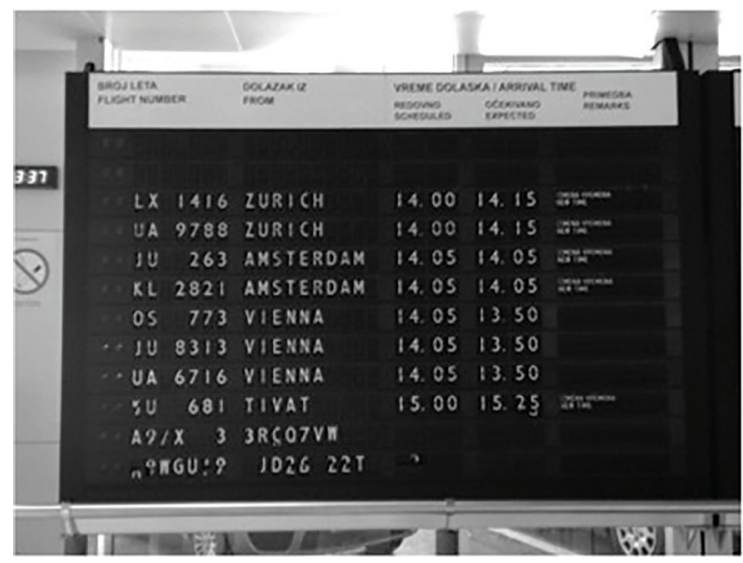

Figure 18. Broken flap display at the airport of Belgrade 
The fact that the era of a possible 'poetics of the mechanical error' is somehow lost forever is emphasized by vintage, hipster, and nostalgic efforts at recuperation, such as the split flap display reconstituted through Arduino materials and visible in Figure 19. The description of the project is extremely telling of its nostalgic connotations:

With three-letter abbreviations like BRB, LOL, OMG and SMH commonplace in today's smartphone and online conversations, the Maker decided to bring text and animation back to its mechanical roots with his own split flap display. As you can see in the video below, an arcade button under each frame enables him to cycle through all 26 letters to spell out his thoughts in acronym form, while a fourth frame reveals an animation of the very first cat video (by Eadweard Muybridge). (Available at https:// blog.arduino.cc/2016/07/15/omg-this-diy-split-flap-display-is-awesome/)

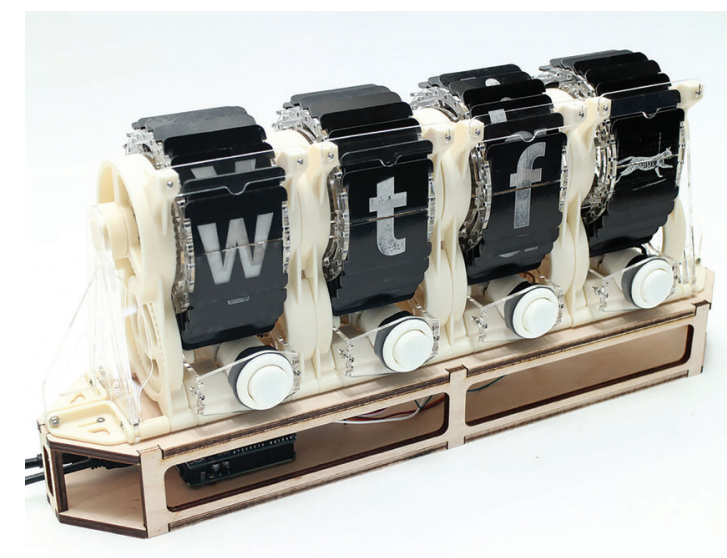

Figure 19. Arduino split flap display

\section{Conclusion: from demosaicing to pixilation}

Are there any chances to turn LCD screens and, more generally, visual digital technology into a matrix for aesthetic effects at least reminiscent of those induced by more traditional 'raster surfaces', such as rugs or mosaics? Although the pixels of LCD screens can potentially malfunction and misfire, as in flickering, such a possibility is so deeply embedded in sophisticated technology that it can rarely give rise to unpredictable aesthetic effects. A very interesting term in digital screen technology is "demosaicing" (also de-mosaicing, demosaicking or debayering), an operation that, through appropriate algorithms, reconstructs a full color image from the incomplete color sample outputs of an image sensor overlaid with a color filter array (CFA). Demosaicising is the ontological and aesthetic opposite of the mosaic: whereas in a 
Byzantine mosaic the tiles contribute to the splendor of the image but, at the same time, do not entirely melt into it - their irreducible singularity farther enhancing the golden vibration of the whole -, in a demosaicised digital image the multitudinous matrix of the screen is concealed behind a numeric, impenetrable smoothness.

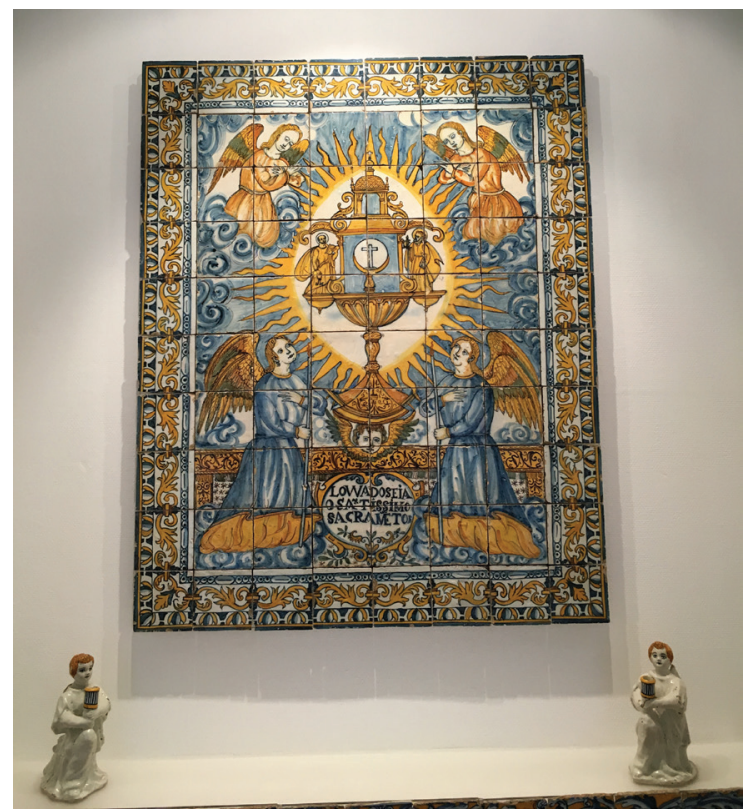

Figure 20. Late $17^{\text {th }}$ century azulejos: the adoration of the Eucharist; Lisbon: National Museum of Azulejos

Figure 20 reproduces the photograph of a splendid Adoration of the Eucharist composed of Portuguese azulejos; one can admire it at the National Museum of Azulejos in Lisbon. In this and similar images, the tiled fragmentation of the image is not an obstacle to its visual composition, but underlays it without ever completely disappearing, so conferring to it a sort of geometric subtext, a connotation of order and regularity (Marks 2010). The gaze enjoys recomposing the image from the single tiles, and yet the singularity of the tiles does not vanish in this visual exercise of reconstitution of the image, since they continue to geometrically vibrate under the figurative surface of the representation. This effect, which turns the composition of azulejos into an effort of creativity non-dissimilar from that which nature deploys in a flowerbed, is completely absent in a correctly functioning LCD screen.

We are more and more surrounded by pixels. Yet, the only possibility for this digital environment to resemble a human fabric of singularities and order, regularities and imperfection, would be through delving into the deep technology of the current displaying matrixes so as to introduce therein a dynamic of pixelation. In filmmaking, pixelation is a technique, dating from the beginnings of cinema, in which live actors are posed frame by frame and photographed to 
create stop-motion animation. The word derives from an archaic British word meaning "possession by spirits" (pixies) (then used to describe the animation process since the early 1950s).

Animating a digital image means turning the subservient logics of the pixels into the semiotics of the pixies, that is, elements that cease to be such for, possessed by mysterious forces, they reclaim the freedom of the singular for the collective project of the digital image, placing it in the subservience of its eternal transmission and marketing allure. Pixies, not pixels, are the equivalent of the unpredictable agencies that move creativity in nature, and that the human species has sought for millennia to reproduce in the paradoxical weaving of its visual artifacts. Forgetting about the beauty of a constantly reemerging imperfection leads to a domain of the cold displaying surface, to the imposition of a framework that is destined to remain inexorably empty.

\section{REFERENCES}

Bahamón, Alejandro and Patricia Pérez 2008. Inspired by Nature: Minerals: The Building/Geology Connection. New York: W.W. Norton.

Calabrese, Omar 2003. Semiotic Aspects of Art History: Semiotics of the Fine Arts. In: Posner,

Roland, Klaus Robering, and Thomas A. Sebeok (eds.) 1997-2004. Semiotik: ein Handbuch zu den zeichentheoretischen Grundlagen von Natur und Kultur, 4 vols. Berlin and New York: Walter de Gruyter, vol. 3, section 14, 3212-33.

Castelli, Richard and Berliner Festspiele, Martin-Gropius-Bau (eds.) 2007. Vom Funken zum

Pixel: Kunst + Neue Medien. Berlin: Berliner Festspiele; Nicolai.

Eco, Umberto 1976. A Theory of Semiotics. Bloomington: Indiana University Press.

Field, Mike and Martin Golubitsky 1992. Symmetry in Chaos: A Search for Pattern in Mathematics, Art, and Nature. Oxford and New York: Oxford University Press.

Girard, René 1977. Violence and the Sacred. Baltimore, MD: Johns Hopkins University Press.

Graw, Isabelle and Ewa Lajer-Burcharth (eds.) 2016. Painting beyond Itself: The Medium in the Post-Medium Condition. Berlin: Sternberg Press.

Greimas, Algirdas Julien 1987. De l'Imperfection. Périgueux: P. Fanlac

Hagener, Malte and Vinzenz Hediger (eds.) 2015. Medienkultur und Bildung: Ästhetische Erziehung im Zeitalter digitaler Netzwerke. Frankfurt and New York: Campus Verlag.

Kandinsky, Wassily 1926. Punkt und Linie zu Fläche: Beitrag zur Analyse der malerischen Elemente. Munich: Albert Langen.

Marks, Laura U. 2010. Enfoldment and Infinity: An Islamic Genealogy of New Media Art. Cambridge, Mass.: MIT Press. 
Marrone, Gianfranco 1990. Cinque interventi tra estetica e semiotica. Palermo: Guida.

Marrone, Gianfranco (ed.) 1995. Sensi e discorso: l'estetica nella semiotica. Bologna: Progetto Leonardo.

Matrix, Sidney Eve 2006. Cyberpop: Digital Lifestyles and Commodity Culture. New York: Routledge.

Mitchell, William J. 2005. Placing Words: Symbols, Space, and the City. Cambridge, Mass.: MIT Press. Pareyson, Luigi 2013. Truth and Interpretation Foreword by Gianni Vattimo, translated and with an introduction by Robert T. Valgenti; revised and edited by Silvia Benso. Albany, NJ: State University of New York Press.

Paul, Gerhard 2016. Das visuelle Zeitalter: Punkt und Pixel. Göttingen: Wallstein Verlag.

Poole, Matthew and Manuel Shvartzberg 2015. The Politics of Parametricism: Digital Technologies in Architecture. London and New York: Bloomsbury Academic.

Romero, Juan and Penousal Machado (eds.) 2008. The Art of Artificial Evolution: A Handbook on Evolutionary Art and Music. Berlin and New York: Springer.

Spieler, Reinhard and Barbara J. Scheuermann (eds.) 2012. Punkt.Systeme: Vom Pointillismus zum Pixel. Heidelberg: Kehrer, 2012.

Tao, Terence 2008. Structure and Randomness: Pages from Year One of a Mathematical Blog. Providence, RI: American Mathematical Society.

Uffelen, Chris van 2009. Fine Fabric: Delicate Materials for Architecture and Interior Design. Berlin: Braun, London: Thames \& Hudson.

Massimo Leone is Professor of Semiotics at the Department of Philosophy at University of Turin, Director of the MA Program in Communication Studies, Editor-in-Chief of Lexia (SCOPUS)

Email: massimo.leone@unito.it 